\title{
A Portable Environmental Parameter Monitor Based on STM32
}

\author{
Pingchuan Zhang, Jie Liu and Sa Zhang \\ School of Information Engineering, Henan Institute of Science and Technology, Xinxiang 453003, \\ China \\ Received: May 11, 2020. Revised: June 19, 2020. Accepted: July 3, 2020. Published: July 20, 2020.
}

\begin{abstract}
In this paper, a portable monitoring system with environmental parameters as breakthrough point is proposed, and focuses on the research and development of a portable instrument for monitoring the quality of indoor environmental parameters. This paper studies and designs a portable environment parameter detector based on STM32 development board, which collects monitoring data through external sensors. Write your own based on STM32 platform of data acquisition and processing procedures, the successful implementation of the, temperature, humidity, formaldehyde, CO, real-time monitoring of environmental parameters such as methane, and through the LCD screen display in real time. Testing environment parameter meter, real-time display of the corresponding data and collecting sample data processing analysis of a day, to achieve the desired goal.
\end{abstract}

Keywords-Sensors, Embedded System, Environmental Parameter Monitor, STM32

\section{INTRODUCTION}

A CCORDING to statistics, people spend $80 \%-90 \%$ of their time indoors, so indoor air quality, whether at home or in the office, has a significant impact on people's quality of life, health status and work efficiency [1-2]. Therefore, indoor air quality is very important for human activities, especially with the acceleration of urbanization, people spend more and more time in indoor activities. At the same time, living space and indoor decoration frequency are also increasing. However, the housing decoration materials contain formaldehyde and other harmful substances, which cause environmental and air quality problems, and bring serious damage to countless families, and increasingly attract people's attention [3-5]. According to the statistics of the World Health Organization, more than $30 \%$ of the building's indoor environment in China is polluted by toxic and harmful gases [6]. Indoor pollution has caused a variety of health problems, and these widespread public health problems can lead to respiratory diseases. Long-term inhalation of low-concentration formaldehyde can cause persistent headache, insomnia, weakness, palpitation, weight loss and vomiting, which seriously affects human health and safety. On the other hand, for office workers, some surveys show that good indoor air quality helps to mobilize the enthusiasm of the staff and improve the work efficiency by $2 \%-16 \%$ [6]. If we can get gas concentration data timely and accurately and when the concentration of harmful gases in the environment exceeds the indoor gas standard, an acousto-optic system will start automatically, all of these will provide a comfortable living environment in real-time.

Air pollution monitoring is not a new research area. In [7], the authors proposed a simple module to measure air quality using the CAN protocol. In the case of the sensor's value exceeds the preset value, an alarm will be triggered and the ventilator will be opened. In [8], a ZigBee WSN-based system for monitoring indoor air quality was implemented by using CC2430 chip to sense temperature, relative humidity and $\mathrm{CO} 2$. In [9], the low-cost tin oxide semiconductor sensor was used to design a smart distributed system for measuring indoor air quality in the health center, which mainly aims at the measurement of indoor temperature, humidity, concentrations of $\mathrm{CO} 2$ and $\mathrm{CO}$, pays little attention to the monitoring of suspended particles indoors. A low cost equipped with GPRS-Sensors monitoring systems was proposed in [10], and the advanced information technology enables us to capture periodic (daily, hourly or even more frequent) records of air pollution levels. The accumulated data are sizeable and useful for analyzing the impact on public health. In paper [11], a novel battery-free sensor module to monitor indoor air quality was proposed, which can realize real-time monitoring and alarm of indoor air quality without extra battery.

To sum up, indoor air quality at home or in the office has great effects on people's quality of life, health status and work efficiency. Therefore, people need to further understand the living and working environments. There is a need for easy-to-use indoor environment monitoring instruments, and users only need to power on to monitor the environmental parameters. The corresponding real-time data can be displayed on the screen for analysis. Environment monitoring should have the sensitive signal alarm function and send alarm when harmful gases such as formaldehyde, $\mathrm{CO}$ are excessive to human body.

In this paper, a portable environmental parameter detector is 
designed. The monitor can measure a variety of environmental indicators. It adopts modular design and is convenient to add other environmental sensors. The data can be displayed on LCD screen in real-time, and the alarm function can be executed.

\section{SYSTEM ARCHITECTURE DESIGN}

\section{A. Demand analysis}

According to the monitoring requirements of various gases, temperature and humidity, we designed a portable, light, real-time and accurate monitor for various environmental parameters, including temperature, humidity, formaldehyde, methane, and $\mathrm{CO}$. The monitor will generate an alarm signal when a target exceeds the standard. Therefore, it can easily measure various harmful gases and provide the real-time alarm function, and it has the advantages of small volume, easy to carry and use. It also has a real-time display function and can output the parameters on the LCD screen.

Table 1 shows the technical indexes of the portable environmental parameter monitor. The standard value of formaldehyde is in accordance with the "Indoor Air Quality Standards", and other items are based on experience.

Table 1. The technical indexes of the monitor

\begin{tabular}{|c|c|c|c|c|}
\hline Name & Range & Resolution & Overstep & Unit \\
\hline CO & $10-1000$ & 1 & 20 & $\mathrm{mg} / \mathrm{m}^{3}$ \\
\hline CH4 & $10-10000$ & 1 & 100 & $\mathrm{mg} / \mathrm{m}^{3}$ \\
\hline HCHO & $0-5$ & 0.01 & 0.1 & $\mathrm{mg} / \mathrm{m}^{3}$ \\
\hline Temperature & $0-50$ & 1 & 30 & ${ }^{\circ} \mathrm{C}$ \\
\hline Humidity & $20-90$ & 1 & 70 & $\% \mathrm{RH}$ \\
\hline
\end{tabular}

\section{B. Design of monitor system}

According to the design requirements, the overall structure of the system is divided into four modules, as shown in Figure 1.

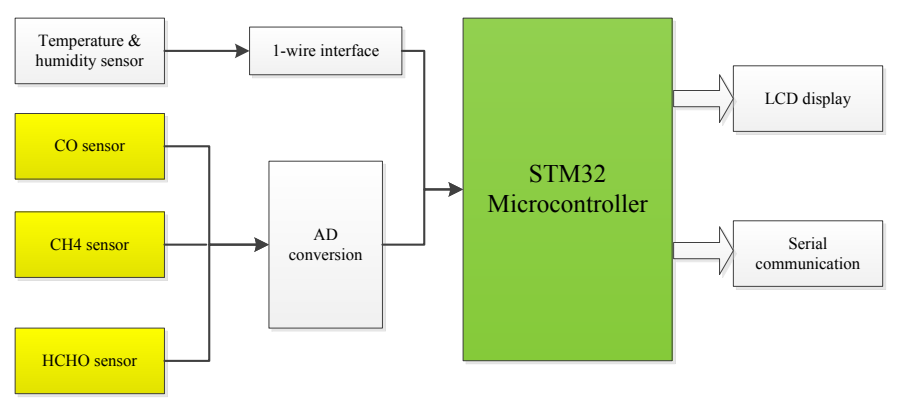

Fig.1. Structure of monitoring system

(1) Data acquisition module: This module is the most important one, as it is the basis of all the work. This module is divided into two parts. The first part is an STM32 built-in analog-to-digital (A/D) conversion channel, which has three gas sensors to sample formaldehyde, methane, and $\mathrm{CO}$ at an interval of $5 \mathrm{~s}$. The second part is the DHT11 temperature and humidity sensor output single bus, which directly obtains digital signals, rather than through the $\mathrm{A} / \mathrm{D}$ conversion.

(2) Process control and data processing module: This module is the core part of the monitor, and its functions include the data conversion and processing, initialization of various hardware systems, and initialization of LCD display module.

(3) LCD display module: This module is mainly responsible for monitoring and displaying the data processed on the LCD screen, and displaying the real-time clock information.

(4) Data output module: This module is responsible for transmitting the real-time monitoring data to the computer through the serial port, and thus facilitates data analysis.

\section{DESIGN OF SYSTEM HARDWARE}

\section{A. Temperature and humidity sensor module}

The DHT11 sensor is a digital sensor integrated with temperature and humidity, and the sensor with high accuracy and stability mainly collects temperature and humidity of the digital monitoring system. DHT11 can use simple single bus communication and only requires a single $\mathrm{I} / \mathrm{O}$ port. The humidity and temperature data of the internal sensor have 4 bytes in total, plus a total of 40 checks are transmitted to the microcontroller at one-time through a single bus. Therefore, the product is superior with high response speed, anti-interference ability, pure digital signal output, and single-wire bus interface, which makes the overall structure of the sensor simpler and easier to use.

The connection of DHT11 digital humidity and temperature sensor is very simple, with totally four pins of ground pin, empty pin, signal output pin, and power pin from left to right. According to the order of the pins, the sensor is connected to the single chip microcomputer. In order to improve the stability, we recommend connecting a $4.7 \mathrm{~K}$ pull-up resistor between the signal output pin and power pin. The diagram of the DHT11 hardware interface circuit is shown in Figure 2. The performance index and characteristics of the DHT11 temperature and humidity sensor are shown in Table 2. 


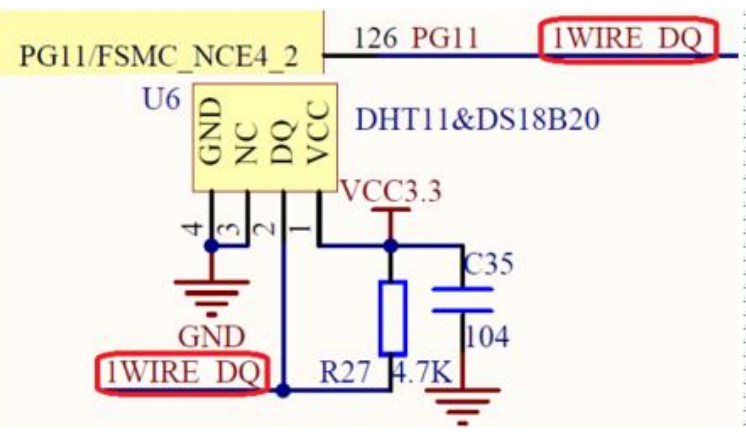

Fig.2. Circuit of temperature and humidity sensor

Table 2. Performance of the temperature and humidity sensor

\begin{tabular}{|c|c|c|}
\hline No. & Index & Property \\
\hline 1 & Voltage & $3.5-5.5 \mathrm{v}$ \\
\hline 2 & Working current & $0.5 \mathrm{~mA}$ \\
\hline 3 & Humidity measurement range & $20-90 \% \mathrm{RH}$ \\
\hline 4 & Temperature measurement range & $0-50^{\circ} \mathrm{C}$ \\
\hline 5 & Resolution of humidity & $1 \% \mathrm{RH}$ \\
\hline 6 & Resolution of temperature & $1{ }^{\circ} \mathrm{C}$ \\
\hline 7 & Sampling period & $1 \mathrm{~s}$ \\
\hline 9 & Transmit mode & $1-$ wire \\
\hline
\end{tabular}

\section{B. CO sensor module}

The system uses an MQ-7 CO sensor, the CO-sensitive MQ-7 sensor is a gas sensor used in low-cost fields, and the material of the internal gas sensor is stannic oxide $(\mathrm{SnO} 2)$. This material has very low conductivity in the air, but the conductivity increases with the concentration of $\mathrm{CO}$ in the air. According to the rules, the change of conductivity can be converted into the output signal corresponding to the gas concentration by using a simple circuit. Therefore, the CO content in the air can be measured.

This design uses the MQ-7 analog output, which accesses to the A/D of the STM32 conversion interface to scan the input. The analog output of MQ-7 is 0-5 V and the A/D input range of STM32 is 0-3.3 V, so the output voltage should be processed, and the output voltage is reduced to $0-2.5 \mathrm{~V}$. The interface circuit diagram of the $\mathrm{CO}$ sensor is shown in Figure 3, and the buck circuit is shown in Figure 4.

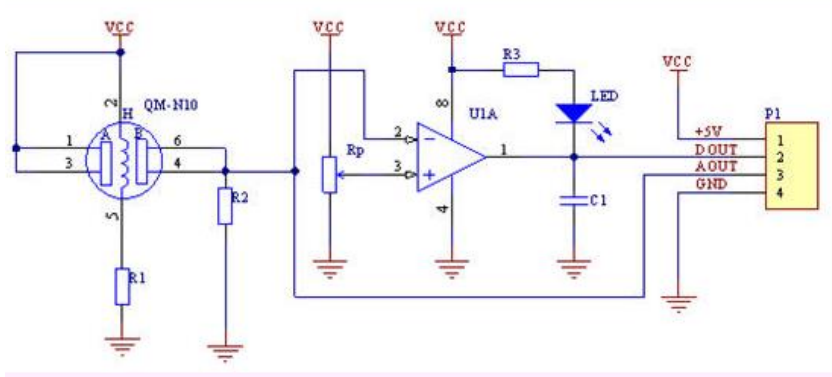

Fig.3. Sensor circuit diagram of CO

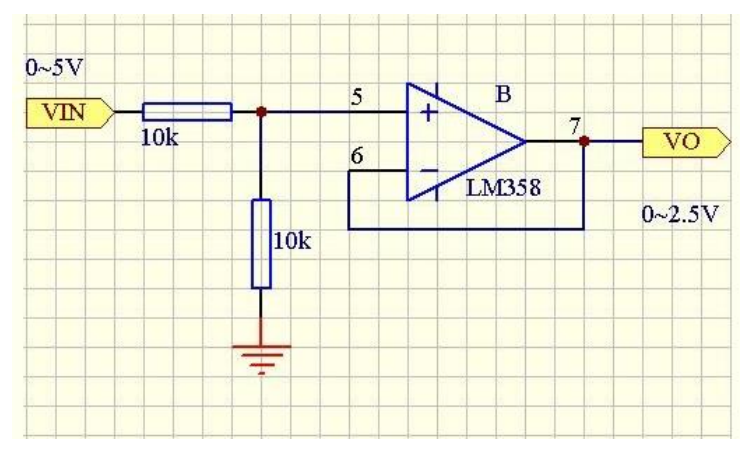

Fig.4. The carbon monoxide sensor circuit

\section{CH4 sensor module}

MQ-4 sensor has high sensitivity to methane, and its conductivity is low in clean air, but when methane exists in air, its conductivity increases with the increase of methane concentration, which is similar to MQ-7 sensor. This design uses the MQ-4 analog output, which accesses to the A/D of the STM32 conversion interface to scan the input. The circuit is the same as CO sensor, as shown in Fig 3 and Fig 4.

\section{HCHO sensor module}

The system selects a ZH08-HCHO formaldehyde module, which is a general electrochemical formaldehyde sensor. This module selectively detects formaldehyde in the air and it has a temperature sensor, which can compensate temperature to achieve higher accuracy. ZH08-HCHO is a common gas module designed and manufactured by combining the mature electrochemical detection technology and excellent circuit design. Since the system adopts A/D conversion input, the output of the sensor is set as 0.4-2.0 V. Table 3 shows the technical indicators of the sensor.

Table 3. Performance of the HCHO sensor

\begin{tabular}{|c|c|c|}
\hline No. & Index & Property \\
\hline 1 & Data output & $0.4-2 \mathrm{~V}$ \\
\hline 2 & Working voltage & $3.7-9 \mathrm{~V}$ \\
\hline 3 & preheating time & Less than 3 minutes \\
\hline
\end{tabular}




\begin{tabular}{|c|c|c|}
\hline 4 & response time & Less than 60 seconds \\
\hline 5 & recovery time & Less than 60 seconds \\
\hline 6 & Range & $0-5 \mathrm{ppm}$ \\
\hline 7 & Resolution & Less than $0.01 \mathrm{ppm}$ \\
\hline 8 & Working temperature & $0-50^{\circ} \mathrm{C}$ \\
\hline
\end{tabular}

\section{DESIGN OF SYSTEM SOFTWARE}

\section{A. Design of system overall program}

The main program flow of this system is elaborated below. First, the first step calls the initialization program, including the initialization of LCD, light emitting diode (LED), analog-to-digital conversion (ADC), direct memory access (DMA), serial port, and real-time clock (RTC). The second step is self-check, which checks whether the RTC and DHT11 hardware are initialized successfully; if not successful, exit. After that, the system displays clock and some fixed signs, such as temperature and humidity. Next, data is sampled and processed every 5 seconds controlled by the clock control system. The real-time data is displayed on the LCD screen. Then it checks whether the data exceeds the standard alarm. Next, data will be transmitted to the computer through the serial data every 5 minutes, followed by data test. Infinite loop operation is run under normal circumstances. The system overall program flow chart is shown in Figure 5.

\section{B. Design of data acquisition program}

The system uses the rules of $\mathrm{A} / \mathrm{D}$ acquisition 1,2 and 3 channels as input channels, which correspond to the $\mathrm{I} / \mathrm{O}$ interfaces of PA0, PA1 and PA2 in scanning mode. Then in the cycle scan mode, the DMA mode is used to input data directly to the static random access memory (SRAM), which reduces the CPU utilization and improves the efficiency of the system. The flow chart of sampling procedure is shown in Figure 6.

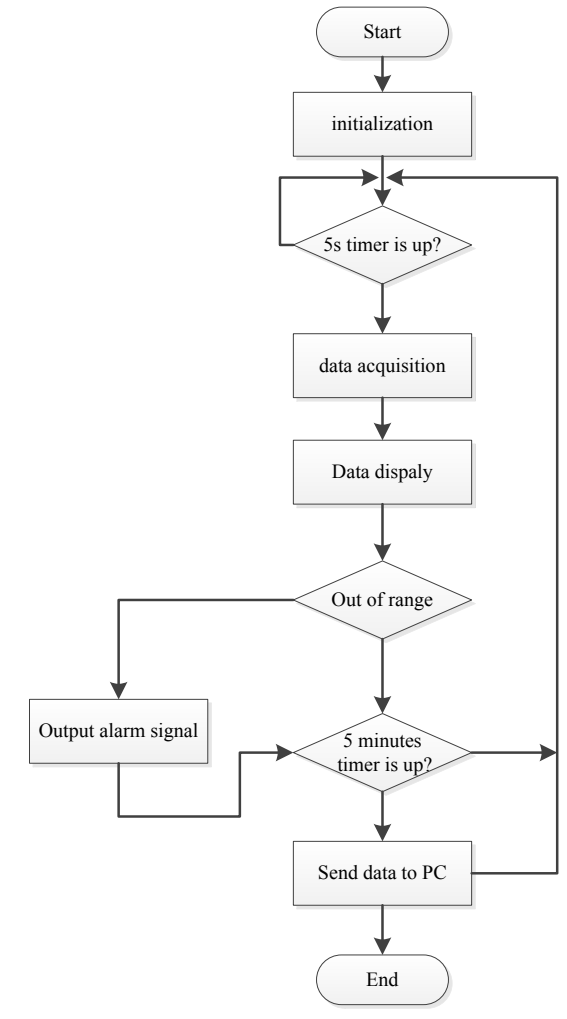

Fig.5. System overall program flow chart

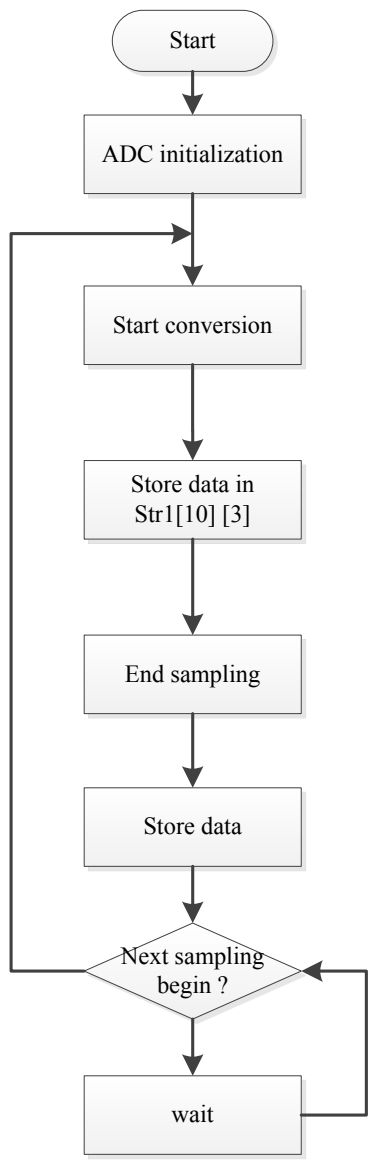

Fig.6. A/D sampling flow chart 
In the sampling process, first, $\mathrm{ADC}$, the configuration of input channel and conversion time, the sampling time of 239 cycles are set to ensure the sampling accuracy. Second, the DMA channel is configured. Using this channel input, the input and output for the target address are configured, the size of the input buffer of the sampling frequency is multiplied by the sampling channel and the system setting buffer, and the goal is to scan 10 sampling cycles. Then the average value is calculated. Third, the A/D conversion is started. Fourth, data is stored in the array Str1 through DMA. Fifth, the A/D conversion is closed. Sixth, the data stored in $\operatorname{Str} 1$ is transferred to the corresponding data storage unit. Seventh, judge whether the next conversion time is reached; if yes, $\mathrm{A} / \mathrm{D}$ conversion is opened again, and the sampling process is repeated; otherwise, the sampling process is repeated until the next conversion.

\section{Design of LCD driver}

The system controls the thin film transistor (TFT)-LCD for display through STM 32 common I/O port analog 8080 bus, and the ILI9341 controller is its driver chip. The read/write (RD/WR) process of 8080 parallel port module: first, according to read/write data types, set the RS as high (data read/write) / low (command read/write), and then select pull low chip and ILI9341. Then we set RD/WR according to the read data or write data:

(1) read data: data on the rising edge of $\mathrm{RD}(\mathrm{D}[15: 0])$;

(2) write data: in the rising edge of WR, data is written into the ILI9341.

The process of using LCD is simple, as shown in the flow chart in Figure 7. Hard reset and initialization sequence only need to be executed once. The process of drawing points is described as follows: set the coordinates, and write GRAM instructions and color data. Then, the corresponding point of the written color is displayed in the LCD above. In the process of reading points: set the coordinates, and read the GRAM instructions and color data. Then we can get the color of the corresponding point data.

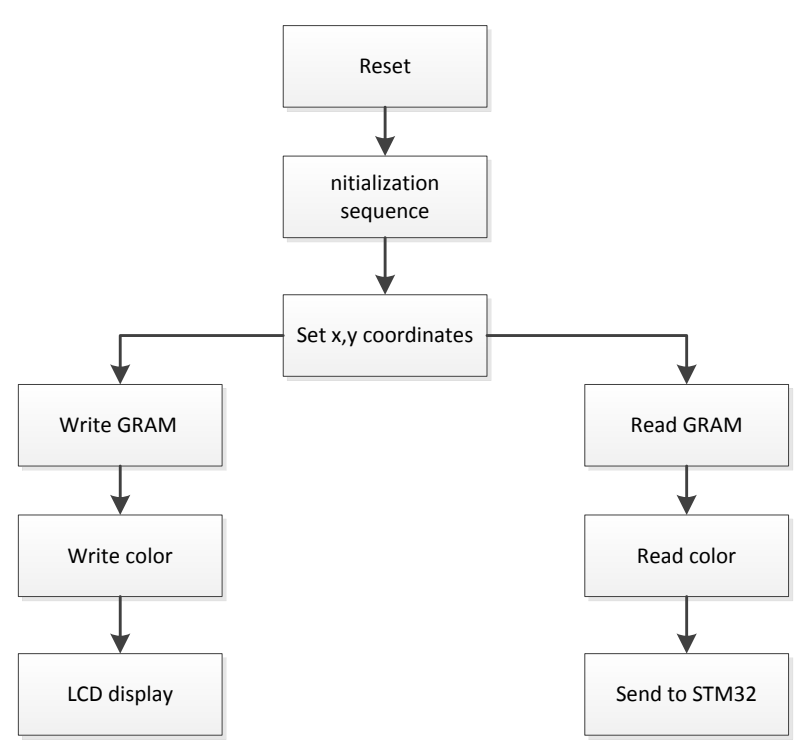

Fig.7. TFT-LCD writing and reading process

\section{System Test}

The system displays real-time data through LCD screen. It can display the measured data of temperature, humidity, $\mathrm{CO}$, methane, and formaldehyde. Due to the limited accuracy of the $\mathrm{CO}$ and methane sensors, we only select and output the integer part. The high precision formaldehyde sensor is selected to output to three decimal places.

We refer to the temperature and humidity sensor calibration standards Methane Oxygen Detection Alarm Instrument Verification Regulation and Light Scattering Digital Dust Testing Instrument Verification Regulation, except for temperature and humidity measurements, the rest cases were tested at the temperature of $20 \pm 3{ }^{\circ} \mathrm{C}$ and relative humidity $\leq$ $60 \%$ of the indoor environment, so as to improve the measurement precision. Three groups of continuous measurement were conducted, and the average value was calculated as the display value of the measuring instrument. The specific test data are shown in Table 4 to Table 7.

Table 4. Temperature measurement data

\begin{tabular}{|c|c|c|c|}
\hline Actual value $/{ }^{\circ} \mathrm{C}$ & $\begin{array}{c}\text { Measurement } \\
\text { value } /{ }^{\circ} \mathrm{C}\end{array}$ & $\begin{array}{c}\text { Absolute } \\
\text { error } /{ }^{\circ} \mathrm{C}\end{array}$ & $\begin{array}{c}\text { relative } \\
\mathrm{error} / \%\end{array}$ \\
\hline 10 & 9.8 & 0.2 & 2 \\
\hline 15 & 14.9 & 0.1 & 0.67 \\
\hline 20 & 20.2 & 0.2 & 1 \\
\hline 25 & 25.1 & 0.1 & 0.4 \\
\hline 30 & 30.3 & 0.3 & 1 \\
\hline
\end{tabular}


Table 5. Humidity measurement data

\begin{tabular}{|c|c|c|c|}
\hline $\begin{array}{c}\text { Actual value } \\
/ \% \mathrm{RH}\end{array}$ & $\begin{array}{c}\text { Measurement value } \\
/ \% \mathrm{RH}\end{array}$ & $\begin{array}{c}\text { Absolute error } \\
/ \% \mathrm{RH}\end{array}$ & relative error $/ \%$ \\
\hline 15 & 15.2 & 0.2 & 1.33 \\
\hline 30 & 30.4 & 0.4 & 1.33 \\
\hline 45 & 45.5 & 0.5 & 1.11 \\
\hline 60 & 60.4 & 0.4 & 0.67 \\
\hline
\end{tabular}

Table 6. HCHO measurement data

\begin{tabular}{|c|c|c|c|}
\hline $\begin{array}{c}\text { Actual value } \\
/ \mathrm{ppm}\end{array}$ & $\begin{array}{c}\text { Measurement value } \\
/ \mathrm{ppm}\end{array}$ & $\begin{array}{c}\text { Absolute error } \\
/ \mathrm{ppm}\end{array}$ & Relative error $/ \%$ \\
\hline 0.5 & 0.51 & 0.01 & 2 \\
\hline 0.8 & 0.78 & 0.02 & 2.5 \\
\hline 1.0 & 1.02 & 0.02 & 2 \\
\hline 2.0 & 1.99 & 0.01 & 0.5 \\
\hline 5.0 & 5.03 & 0.03 & 0.6 \\
\hline
\end{tabular}

Table 7. CH4 measurement data

\begin{tabular}{|c|c|c|c|}
\hline $\begin{array}{c}\text { Actual value } \\
/ \mathrm{ppm}\end{array}$ & $\begin{array}{c}\text { Measurement value } \\
/ \mathrm{ppm}\end{array}$ & $\begin{array}{c}\text { Absolute error } \\
/ \mathrm{ppm}\end{array}$ & $\begin{array}{c}\text { Relative error } \\
/ \%\end{array}$ \\
\hline 0.5 & 0.48 & 0.02 & 4 \\
\hline 0.8 & 0.83 & 0.03 & 3.75 \\
\hline 1.0 & 1.02 & 0.02 & 2 \\
\hline 2.0 & 2.01 & 0.01 & 0.5 \\
\hline 5.0 & 5.02 & 0.02 & 0.4 \\
\hline
\end{tabular}

In Table 4 , the actual indoor temperature values are respectively $10,15,20,25$ and $30^{\circ} \mathrm{C}$. The designed monitor measures each group of data three times, and then the measured temperature values after taking the average value are $9.8,14.9$, $20.2,25.1$ and $30.3^{\circ} \mathrm{C}$, respectively. In Table 5, the actual indoor relative humidity values are respectively $15,30,45$ and $60 \%$ RH. After the same measurement method, the relative humidity values measured by the designed monitor are 15.2, $30.4,45.5$, and $60.4 \% \mathrm{RH}$, respectively. The above data show that the measurement errors of temperature and humidity are both about $1 \%$. In Table 6 , the actual indoor $\mathrm{HCHO}$ values are $0.5,0.8,1.0,2.0,5.0 \mathrm{ppm}$, respectively. The designed monitor measures each group of data three times, and then the measured $\mathrm{HCHO}$ values after taking the average value are respectively $0.51,0.78,1.02,1.99,5.03 \mathrm{ppm}$, respectively. In Table 7 , the actual indoor $\mathrm{CH} 4$ values are 0.5, 0.8, 1.0, 2.0 and 5.0ppm, respectively. After the same measurement method, the $\mathrm{CH} 4$ values measured by the designed monitor are $0.48,0.83,1.02$, 2.01 and $5.02 \mathrm{ppm}$, respectively. For the measurement of gas concentration, the measurement error is relatively large at small gas concentrations, and is low at large gas concentrations. This is mainly due to the detection circuit of the resolution caused by insufficient sensitivity. In summary, a monitor for gas concentration detection in living and working environment is developed here, which meets the measurement requirements.

\section{CONCLUSION}

This paper is focused on the research and development of a portable parameter monitoring instrument for indoor environment. On the platform of an STM32 development board, the monitored data are collected through external sensors. The STM32 platform is used to carry out the program of data acquisition and processing, and successfully monitors environmental parameters of temperature, humidity, formaldehyde, $\mathrm{CO}$, methane in real-time. Real-time data are displayed on the LCD screen. The system is stable and can be used in different indoor places such as family, hospital, and office. Meanwhile, the system adopts the idea of modular design and is superior in transplanting. The scheme is slightly modified and can be used similarly as the function of a monitoring system. The monitor has very high research and application values.

\section{ACKNOWLEDGMENT}

This work was supported in part by Henan Province Department of Science and Technology Research Projects (Granted No.152102210309,1720210250, 182102110294), and Key Scientific Research Project of Henan Provincial Education Department (Granted No.14B120009,18A210014).

\section{REFERENCES}

[1] Orosa J A, Oliveira A C. "Hourly indoor thermal comfort and air quality acceptance with passive climate control methods". Renewable Energy. vol. 34, pp.2735-2742, 2009.

[2] Saha C K, Zhang G, Kai P, et al. "Effects of a partial pit ventilation system on indoor air quality and ammonia emission from a fattening pig room”. Biosystems Engineering. vol. 105, pp.279-287, 2010.

[3] Kim M J, Braatz R D, Kim J T, Yoo C K. "Indoor air quality control for improving passenger health in subway platforms using an outdoor air quality dependent ventilation system". Building and Environment. vol.92, pp. 407-417, 2015.

[4] Asadi E, Da S, Manuel C G, Costa J J. "A systematic indoor air quality audit approach for public buildings". Environmental Monitoring and Assessment. vol.185 pp. 865-875, 2013.

[5] Das P, Shrubsole C, Jones B, et al. "Using probabilistic sampling-based sensitivity analyses for indoor air quality modelling", Building \& Environment. vol.78, pp.171-182, 2014.

[6] Fanger PO. "Perceived air quality, sick building syndrome (SB) symptoms and productivity in an office with two different pollution loads (Published Conference Proceedings References)" . in Proceedings of the 8th International Conference on Indoor Air Quality and Climate-Indoor Air, Edinburgh, 1999, pp.165-179.

[7] PILLAI M A, VEERASINGAM S, YASWANTH S D. "CAN based smart sensor network for indoor air quality monitoring (Published Conference Proceedings References)". In 3rd IEEE International Conference Computer Science and Information Technology (ICCSIT), 2010, pp.456-460.

[8] YU C B, CUI Y Z, ZHANG L et al. ZigBee wireless sensor network in environmental monitoring applications (Published Conference Proceedings References)". In 5th International Conference Wireless Communications Networking and Mobile Computing, 2009, pp.1-5. 
[9] Alhmiedat T. "Low-power environmental monitoring system for zigbee Wireless sensor network". Ksii Transactions on Internet \& Information Systems, vol.11, pp.4781-4803, 2017.

[10] N Kularatna, BH Sudantha. "An environmental air pollution monitoring system based on the IEEE 1451 standard for low cost requirements". IEEE Sensors Journal. vol.8, pp. 415-22, 2008.

[11] Tran T V, Dang N T, Chung W Y. "Battery-free smart-sensor system for real-time indoor air quality monitoring". Sensors \& Actuators B Chemical, vol.248, pp.930-939, 2017.
Creative Commons Attribution License 4.0 (Attribution 4.0 International, CC BY 4.0)

This article is published under the terms of the Creative Commons Attribution License 4.0

https://creativecommons.org/licenses/by/4.0/deed.en_US 